\title{
Mammary Gland Expression of Antibacterial Peptide Genes to Inhibit Bacterial Pathogens Causing Mastitis
}

\author{
J. X. Zhang, S. F. Zhang, T. D. Wang, X. J. Guo, and R. L. Hu' \\ Veterinary Institute, Academy of Military Medical Sciences, 1068 Qinglong Road, Changchun 130062, P.R. China
}

\section{ABSTRACT}

As a step toward prevention of bovine mastitis, a plasmid-mediated gene transfer technique was used to enable mammary cells to synthesize and secrete bovine lactoferricin and bovine tracheal antibacterial peptides. For this purpose, a series of mammary tissue-specific expression vectors, harboring the antibacterial peptide gene, the 5 '-flanking regulation sequence of goat $\beta$-casein, and the bovine growth hormone polyadenylation signal sequence, were constructed using a eukaryotic expression vector pIRES1-neo. The mammary gland tissue-specific expression vector carrying the antimicrobial peptide genes dissolved in physiologic saline was injected directly into the lactating mammary glands of goats. The milk samples after injection were checked by Tricine-SDS-PAGE and bacterium inhibition zone assay. The results of these tests showed that the mammary gland tissue-specific expression vector driven by the goat $\beta$-casein gene promoter could efficiently direct the expression of antibacterial peptides in goat milk; the expression of antibacterial proteins lasted for 3 to $6 \mathrm{~d}$. All of the milk samples collected from the mammary glands that had been injected with different vectors harboring the antibacterial peptide gene(s) exhibited bacteriostatic activity against different bacterial pathogens. These results demonstrated that the mammary gland tissue-specific expression vector could be used to introduce antibacterial peptide gene into the goat mammary gland, enabling secretion of a bioactive form of antibacterial peptide in the milk. This successful expression of antibacterial peptides in goat mammary glands provided a possible method to prevent mastitis in ruminants.

Key words: mastitis, antibacterial peptide, bovine lactoferricin, bovine tracheal antibacterial peptide

\section{INTRODUCTION}

Bovine mastitis is a highly prevalent disease in dairy animals. It places a heavy economic burden on milk

Received April 22, 2007.

Accepted July 19, 2007.

${ }^{1}$ Corresponding author: hurongliang@hotmail.com producers all over the world (Bennett et al., 1999). Mastitis is inflammation of the mammary glands, usually caused by microbial infection. Mastitis-causing pathogens include bacteria and nonbacterial pathogens such as mycoplasms, fungi, yeasts, and Chlamydia (Watts, 1988). Staphylococcus aureus and Escherichia coli are primarily responsible for the bulk of bovine mastitis cases. The classic symptoms of mastitis are inflammation, swelling of the mammary glands, and increase of SCC in milk. It decreases milk production and milk quality and increases herd management cost (Harmon, 1994). Systematic application of antibiotic therapy at dry-off cures a number of infections and plays a role in prevention of new infection during this susceptible period, but problems might occur such as antibiotic residues in the body and potential development of antibiotic resistant bacterial strains in repeated use of antibiotics (Aarestrup et al., 1995; Goñi et al., 2004). Chinese traditional medicines; for example, Diluteyellow crotalaria herb, Tokyo violet herb, Forsythia suspense, Honeysuckle flower, Lion's tooth, Astragali, and Paeoniae rubra have exerted a certain role in mastitis treatment. All of the Chinese traditional medicines combined in a certain prescription exhibit a therapeutic effect on mastitis similar to or, to some extent, lower than antibiotics. However, the dose required is large, and the cost is high (Wang and Zhang, 2004). Related vaccines to mastitis are not in common use due to problems associated with lack of widespread efficiency. Development of new vaccines is under investigation (Castagliuolo et al., 2006).

Antimicrobial peptides (AMP) isolated from many different organisms during the last decades (Zasloff, 2002) have a wide range of antibacterial activities. Most AMP, consisting of less than 50 amino acids, are amphipathic and carry a net positive charge (Van't Hof et al., 2001). Extensive studies on AMP have shown that they exert their action on the cytoplasmic membrane of susceptible microorganisms. Different molecular mechanisms of membrane perturbation by antimicrobial peptides have been proposed (Lohner and Blondelle, 2005). For example, AMP might result in pores on the bacterial membrane by thinning the membrane or destabilizing 
the membrane bilayer (Gazit et al., 1995; Heller et al., 2000). Furthermore, AMP are able to kill microorganisms by inhibition of macromolecule biosynthesis, or by interacting with specific vital components inside the microorganism, or both (Ulvatne et al., 2004).

Bovine lactoferricin (LfcinB) is a peptide released from bovine lactoferrin upon gastric pepsin cleavage (Tomita et al., 1991), it consists of 25 amino acid residues (17-41) from the N-terminal region of lactoferrin, and has a net charge of +8 (Bellamy et al., 1992). This region has a remarkable conformational flexibility that allows it to form an amphipathic structure in solution, which, in turn, can lead to binding of the peptide to the negatively charged membrane of microbial surfaces (Vogel et al., 2002). Bovine lactoferricin has shown the best biological activity, and it possesses the antimicrobial activity against a wide range of microorganisms, including gram-positive and gram-negative bacteria (Bellamy et al., 1992) as well as fungi (Wakabayashi et al., 1999). In addition, the peptide has shown antiviral and antitumor activities (Di Biase et al., 2003). Moreover, the peptide is capable of stimulating the adaptive immune response and has antiinflammatory properties (Feng et al., 2004).

Bovine tracheal antimicrobial peptide (bTAP) is a member of the $\beta$-defensin family of antibiotic peptides found in the cow tracheal mucosa (Selsted et al., 1993). It is a highly abundant cysteine-rich antimicrobial peptide. The bTAP gene expression in bovine airway is inducible by lipopolysaccharide and inflammatory mediators, suggesting that it functions to protect the upper airway from infection (Yarus et al., 1996). This peptide exhibits broad-spectrum activity when assayed in vitro against several different strains of microbes, including some respiratory pathogens (Diamond et al., 1991).

Fan et al. (2004) utilized the adenovirus-mediated gene transfer method to direct expression of lysostaphin and found that the expressed lysostaphin inhibited the growth of Staphylococcus aureus in vitro. Other trials also showed that both viral and nonviral vectors could deliver genes to a variety of sites in different animals and in humans (Archer et al., 1994; Hens et al., 2000). We have attempted to develop an alternative strategy to combat and prevent mastitis originated from bacterial pathogens by enabling the cells of the lactating mammary glands to produce exogenous antibacterial proteins. The prerequisite in application of antibacterial peptides in combating mastitis program is that these peptides should be successfully secreted by the mammary cells and be restricted to the inside of mammary glands. Direct delivery of genes encoding antimicrobial proteins into the mammary gland would be the easiest way. The current study is designed to determine whether this strategy would enable the mammary cells to secrete bioactive antibacterial peptides.

\section{MATERIALS AND METHODS}

\section{Synthesis of Antibacterial Peptide Genes}

Oligonucleotide fragments encoding LfcinB and bTAP were designed according to the primary amino acid sequence of native LfcinB (GenBank Acc. no. L08604) and bTAP (GenBank Acc. no. AF014106), synthesized by Shanghai Sangon Biological Engineering and Technology Service Co. Ltd. (Shanghai, China). The LfcinB- and bTAP-encoding fragments were generated by annealing with one another and then ligation of oligonucleotides, respectively.

Oligonucleotides for LfcinB were

F1: 5'-GCGGCCGCATGAAGCTCTTCGTCCCCGCCC TGCTGTCCCTTGGAGCCCTT

R1: 5'-CTGCCATCGGCGCATTTGAAAGCCAGACAC AGTCCAAGGGCTCCAAGGGACAG

F2: 5'-AAATGCCGCCGATGGCAGTGGAGGATGAA GAAGCTGGGTGCTCCC

R2:5'-GGATCCTTAAAAGGCCCTCCTCACACAGGT GATAGAGGGAGCACCCAGCTTCTT

Oligonucleotides for bTAP were

F1:5'-CCCGGGATGAGGCTCCATCACCTGCTCCTC CTCTCCTCTTCCTGGTCCTGTCTGCTTCCTCA

R1:5'-CTTTATTCCTAACACAGCTTACAGGATTTCC TACTCCTTGAGTAAATCCTGAGGAAGCAGACAGG $\mathrm{AC}$

F2:5'-GCTGTGTTAGGAATAAAGGCATCTGTGTGC CAATCAGGTGTCCTGGAAACATGAAACAGATTGG CACC

R2:5'-TCTAGATTACTTCTTTCTACAGCATTTTACT GCTCTCCCGACACAGGTGCCAATCTGTTTCAT

Oligonucleotides F1 and R1 have an 18-bp complementary overlapping at their $3^{\prime}$-ends. Oligonucleotides F2 and R2 have an 18-bp complementary overlapping at their $3^{\prime}$-ends; R1 and F2 have an 18-bp complementary overlapping at their $5^{\prime}$-ends, respectively, and contain a translation termination codon TAA. To facilitate the cloning, restriction endonuclease recognition sites were added to the 5 '-end and 3 '-end: GCGGCCGC and GGATCC sequences were introduced at the $5^{\prime}$-end and at the $3^{\prime}$-end of the LfcinB oligonucleotides, respectively; CCCGGG and TCTAGA sequences were intro- 
duced at the $5^{\prime}$-end and at the $3^{\prime}$-end of the bTAP oligonucleotides, respectively. An equimolar mixture of the 4 primers was mixed up and annealed with the nicks between $\mathrm{F} 1$ and $\mathrm{R} 1$ and $\mathrm{F} 2$ and $\mathrm{R} 2$ sealed according to the method described elsewhere (Zhu et al., 2002). After amplification, cloning, and sequencing (Shanghai BioAsia Biological Technology Service Co. Ltd., Shanghai, China), the DNA sequences of LfcinB and bTAP were confirmed to be correct.

\section{Construction of Antibacterial Peptide Mammary Gland Tissue-Specific Expression Vector}

Plasmid pIRES1-neo (GenBank Acc. No. U89673; Cat. no. 6060, Clontech Laboratories, Palo Alto, CA) was used as the basal plasmid in vector construction. A mammary-gland-tissue-specific expression vector was constructed by replacing the cytomegalovirus (CMV) promoter sequence of pIRES1-neo with a 1,184 base pairs fragment including the goat $\beta$-casein promoter sequence (GenBank Acc. no. AY311384), resulting in a vector pIbcp-neo, which can direct the expression of a foreign gene in the lactating mammary gland. The LfcinB and the bTAP genes were simultaneously cloned into the vector at the multiple cloning sites and in place of the neomycin phosphotransferase gene, forming $\mathrm{pI}$ bcp-LfcinB/bTAP. The LfcinB or bTAP genes were separately subcloned into pIbcp-neo and the neomycin phosphotransferase gene in pIbcp-neo was excluded, forming pIbcp-LfcinB and pIbcp-bTAP, respectively. The construction methods of the expression vectors and their preparation in large scale followed the standard protocols (Sambrook et al., 1989).

\section{Expression of Antibacterial Peptide in Goat Milk}

Eighteen Shannon goats (Yidong Goat Breeding, Changchun, China) in lactation (3 to $7 \mathrm{~d}$ after parturition) were used for antibacterial peptide expression. They were divided into 3 groups, 6 goats in each group. In group $1,400 \mu \mathrm{g}$ of recombinant plasmid pI-bcpLfcinB/bTAP was dissolved in $5 \mathrm{~mL}$ of physiological saline and injected into the lactating mammary glands of left side using a 6.5-gauge sterile needle. Mammary glands of right side were served as controls and received the same volumes of infusion physiological saline containing the empty plasmid pIbcp-neo. The syringe was gradually withdrawn while the plasmid solution was injected into the tissue to ensure that the plasmid solution was distributed evenly. In group 2 , the same amount of the recombinant plasmids pIbcp-LfcinB was injected into left mammary glands of 6 goats; in group 3 , the recombinant plasmids pIbcp-bTAP were injected into left mammary glands of another 6 goats, and in the meantime the control mammary glands were set to assay the expression pattern and the antibacterial activities of LfcinB and bTAP, respectively. Five-milliliter milk samples were collected immediately before injection and $3 \mathrm{~h}$ after the injection with $3 \mathrm{~h}$ interval for $7 \mathrm{~d}$. The milk was centrifuged at $12,500 \times \mathrm{g}$ for $5 \mathrm{~min}$. The lipid layer and the sediments were discarded, and the intermediate phase was recovered for assay.

\section{Tricine-SDS-PAGE Analysis of Milk Containing the Recombinant Antibacterial Peptide}

The milk containing the antibacterial peptide was centrifuged at $12,500 \times g$ for $15 \mathrm{~min}$ and $20 \mu \mathrm{L}$ of the intermediate phase was removed and mixed with the same volume of loading buffer [50 $\mathrm{m} M$ Tris-HCl $(\mathrm{pH}$ 6.8), $100 \mathrm{mM}$ dithiothreitol, $2 \%$ SDS, $0.1 \%$ bromophenol blue, and $10 \%$ glycerol]. The mixture was boiled for 5 min and allowed to cool to room temperature for $2 \mathrm{~min}$, and then separated on Tricine-SDS-PAGE and stained with Coomassie Brilliant Blue R-250. The method followed the protocols described elsewhere (Sambrook et al., 1989).

\section{Antibacterial Activity Assay of the Recombinant Antibacterial Peptide In Vitro}

The antibacterial activity of the recombinant antibacterial peptides expressed in milk was measured through a bacterium growth inhibition zone assay described elsewhere (Lehrer et al., 1991). Briefly, Staphylococcus aureus ATCC 25923 and Escherichia coli $\mathrm{K}_{12} \mathrm{D}_{31}$ (purchased from Chinese Institute of Veterinary Drug Control) were grown overnight at $37^{\circ} \mathrm{C}$ in $50 \mathrm{~mL}$ of Luria broth $(\mathbf{L B})$ medium, $500 \mu \mathrm{L}$ of culture was inoculated into $50 \mathrm{~mL}$ of fresh $\mathrm{LB}$ medium and incubated at $37^{\circ} \mathrm{C}$ for additional $3 \mathrm{~h}$. The bacteria were centrifuged at $900 \times g$ for $5 \mathrm{~min}$ at $4^{\circ} \mathrm{C}$, washed, and resuspended in $10 \mathrm{~mL}$ of cold PBS to reach an optimal density of $5 \times 10^{7} \mathrm{cfu} / \mathrm{mL}$ (OD $\approx 0.2$ ). Then, $20 \mu \mathrm{L}$ bacterial suspension $\left(1 \times 10^{6}\right.$ bacterial cells $)$ was inoculated into $10 \mathrm{~mL}$ of prewarmed $\left(45^{\circ} \mathrm{C}\right) \mathrm{LB}$ medium containing $1 \%$ (wt/vol) low electroendosmosis-type agarose (SigmaAldrich, St. Louis, MO). After vortex, agarose containing the bacteria was poured onto a $100 \times 50 \mathrm{~mm}$ transparent plastic plate. After set, wells were made in the solidified agarose plate using a sterilized punch. Twenty-microliters milk samples were added to the wells, serving as the treatment and the control, respectively. The plate was incubated at $37^{\circ} \mathrm{C}$ for $16 \mathrm{~h}$.

Blood, urine, and saliva samples were also collected within $48 \mathrm{~h}$ after the recombinant plasmid injection; meanwhile, the milk samples were collected as expression positive controls. All the samples were checked 


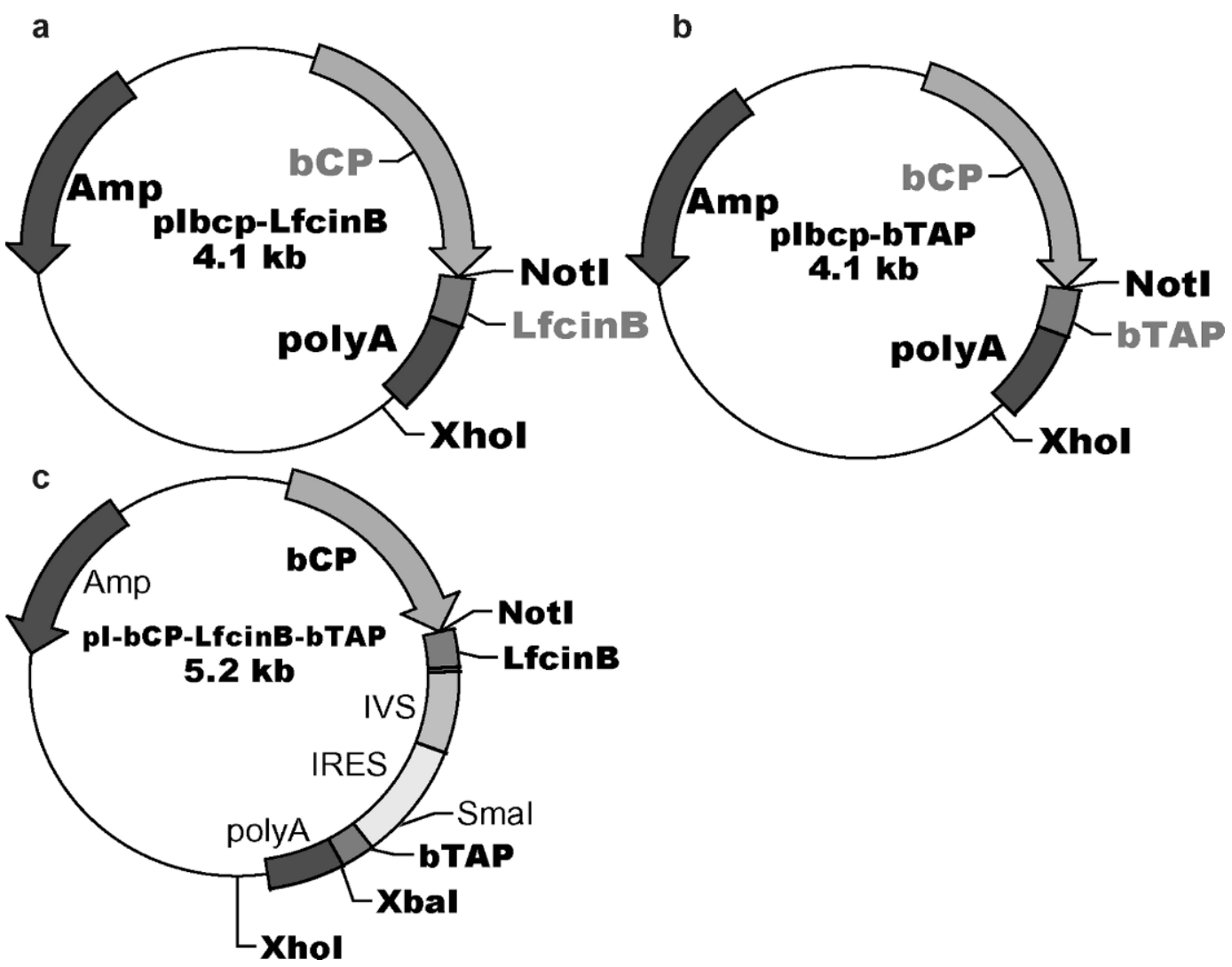

Figure 1. Construction of mammary gland tissue-specific expression vector harboring different antibacterial peptide genes. (a) The bovine lactoferricin (LfcinB) gene was inserted in the mammary gland tissue-specific expression vector; (b) the bovine tracheal antimicrobial peptide (bTAP) gene was inserted in the mammary gland tissue-specific expression vector; (c) the LfcinB and the bTAP genes were inserted at the multiple cloning sites and the site of the neomycin phosphotransferase gene, respectively. The cytomegalovirus (CMV) promoter in pIRES1neo was replaced by goat $\beta$-casein promoter, Amp stands for ampicillin resistance gene, IVS (intervening sequence) is a synthetic intron, IRES stands for internal ribosome entry site of the encephalomyocarditis virus, neo is the abbreviation of neomycin phosphotransferase gene, and polyA is a fragment of the bovine growth hormone poly(A) signal.

for antibacterial activity against Staphylococcus aureus ATCC 25923 by the method described as above.

\section{RESULTS}

\section{Construction of Antibacterial Peptide Expression Vectors}

The construction strategies of the 2 antibacterial peptide genes cloned into mammary gland tissue-specific expression vector are shown in Figure $1 \mathrm{a}, \mathrm{b}$, and c. The plasmid pIRES1-neo used in this study is a commercially sold plasmid. Replacing the CMV promoter in pIRES1-neo by a caprine $\beta$-casein promoter enables the gene of interest downstream to be expressed in a mammary-gland-specific way at lactating stage, which indicates that the expression of the antibacterial peptides was under the control of the goat $\beta$-casein promoter. The expression vector contains the internal ribosome entry site of the encephalomyocarditis virus, which per- mits the translation of 2 open reading frames from 1 messenger RNA. Meanwhile, the neomycin phosphotransferase gene in the vector was replaced by another antibacterial peptide gene. Therefore the genes that could be expressed in the mammary gland tissue-specific expression vector are the antibacterial peptide genes, ensuring that the antibacterial peptides are the only foreign proteins in the milk.

\section{Antibacterial Peptide Expression in Goat Milk}

After plasmid injection into the mammary glands, the foreign antibacterial peptides were expressed and secreted into the goat milk, whereas in different milk controls no foreign protein expression could be observed (Figure 2a, b, and c). The amount of the expressed antibacterial peptides was 0.42 to $0.65 \%$ among different milk samples by scanning analysis. But the difference of antibacterial peptides expression in the selected 
a.

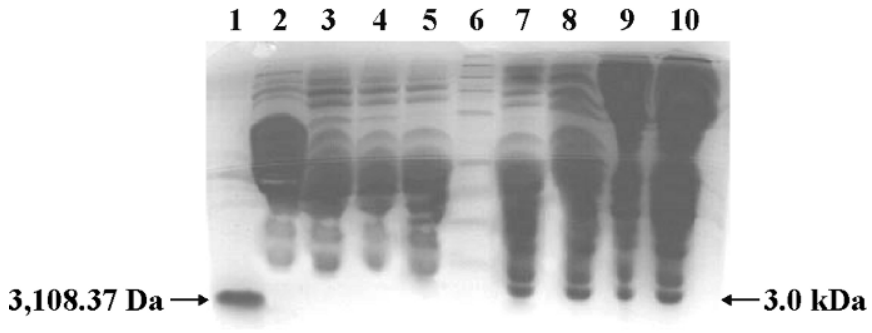

b.

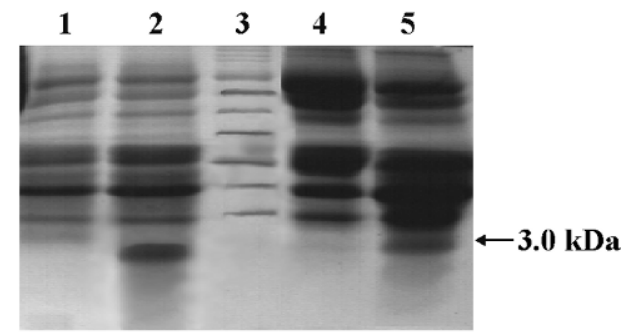

c.

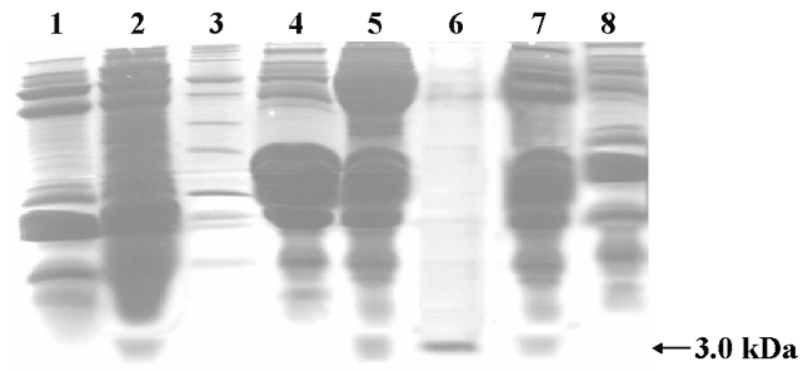

Figure 2. Tricine-SDS-PAGE analysis of bovine lactoferricin (LfcinB), bovine tracheal antimicrobial peptide (bTAP), and LfcinB/ bTAP in goat milk samples. In Figure 2a, lane 1 is thymic peptide $\alpha 1$, the molecular weight of which is $3,108.37 \mathrm{Da}$; lanes $2,3,4$, and 5 are milk samples from different individuals collected before infusion of the expression plasmids; lanes 7 and 8 are goat milk samples from 2 mammary glands after injection of the recombinant plasmids containing LfcinB gene; lanes 9 and 10 are goat milk samples from 2 mammary glands after injection of the recombinant plasmids containing bTAP gene. In Figure 2b, lanes 1 and 4 are milk samples from different individuals before infusion of the recombinant plasmids; lanes 2 and 5 are goat milk samples from 2 mammary glands after injection of the recombinant plasmid containing the LfcinB/ bTAP gene. In Figure 2c, lanes 1, 4, and 8 are milk samples from different individuals after injection of the control plasmid pIbcp-neo; lane 6 is thymic peptide $\alpha 1$; lanes 2,5 , and 7 are milk samples from different individuals after injection of the recombinant plasmids containing LfcinB, bTAP, and LfcinB/bTAP genes, respectively. Lane 6 in figure $2 \mathrm{a}$, lane 3 in figure $2 \mathrm{~b}$, and lane 3 in Figure 2c are low molecular weight protein markers, among which the minimum molecular weight is $7.0 \mathrm{kDa}$. The molecular weight of the 2 recombinant antibacterial peptides in milk samples is shown to be around $3.0 \mathrm{kDa}$.

goats was not significant. The molecular weight of the recombinant antibacterial peptide is around $3.0 \mathrm{kDa}$, compared with low molecular weight protein markers (Figure 2a, b, and c), which was similar to that calculated according to their amino acid compositions.

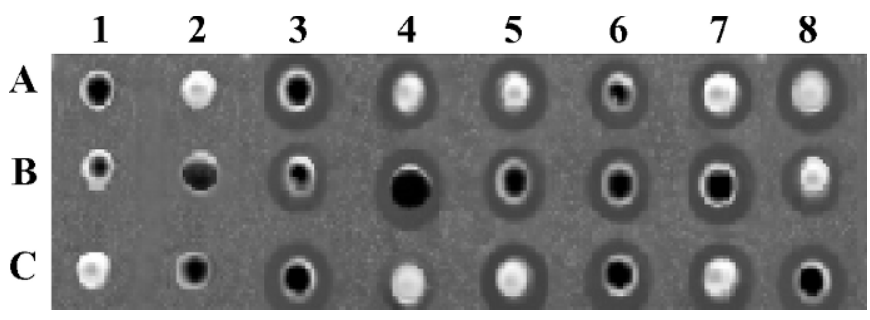

Figure 3. The antimicrobial activity of the expressed bovine lactoferricin (LfcinB), LfcinB/bTAP, and bovine tracheal antimicrobial peptide (bTAP) against Escherichia coli $\mathrm{K}_{12} \mathrm{D}_{31}$. A1, B1, and $\mathrm{C} 1$ are negative milk controls; $\mathrm{A} 2, \mathrm{~B} 2$, and $\mathrm{C} 2$ are empty plasmid milk controls; A3-8, B3-8, and C3-8 are milk samples collected at 3, 6, 9, 12, 24, and $72 \mathrm{~h}$ after the plasmid injection. Row A samples are milk containing the expressed LfcinB; row B samples are milk containing the expressed LfcinB/bTAP; and row $\mathrm{C}$ samples are milk containing the bTAP (Note: The white dots and rings are lipoprotein derived from the milk. The black dots are black background. The gray color was formed by growth of Escherichia coli $\mathrm{K}_{12} \mathrm{D}_{31}$ on the agarose plate. The semitransparent rings are the bacterium growth inhibition zone).

\section{The In Vitro Antibacterial Activity of Milk Containing the Recombinant Antibacterial Peptides}

The antibacterial activity of the antibacterial peptides expressed in the milk was demonstrated in bacterial growth inhibition zone assay (Figures 3 and 4). Both LfcinB and bTAP expression milk samples showed inhibitory activity against Staphylococcus aureus and Escherichia coli, which indicates a broad spectrum of antibacterial activity at least including the gram-negative and gram-positive bacteria. However, the milk from all the negative controls did not show such activity. This demonstrated that the recombinant antibacterial peptides could be efficiently expressed in the lactating mammary glands and their antibacterial activities be displayed in the milk.

The expression persistency of antibacterial peptides was proved to be about $6 \mathrm{~d}$ by both Tricine-SDS-PAGE

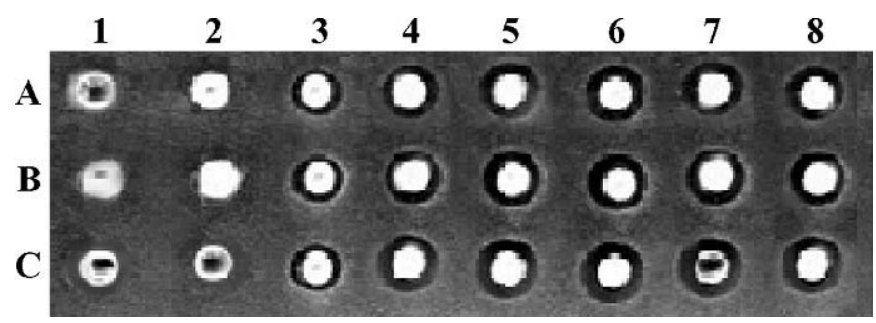

Figure 4. The antimicrobial activity of recombinant bovine lactoferricin (LfcinB), LfcinB/bTAP, and bovine tracheal antimicrobial peptide (bTAP) against Staphylococcus aureus ATCC 25923. A1, B1, and $\mathrm{C} 1$ are negative milk controls; $\mathrm{A} 2, \mathrm{~B} 2$, and $\mathrm{C} 2$ are empty plasmid milk controls; A3-8, B3-8, and C3-8 are milk samples collected at $3,6,9,12,24$, and $72 \mathrm{~h}$ after the plasmid injection. Row A samples are milk containing the expressed LfcinB; row B samples are milk containing the expressed LfcinB/bTAP; and row $\mathrm{C}$ samples are milk containing the bTAP. 


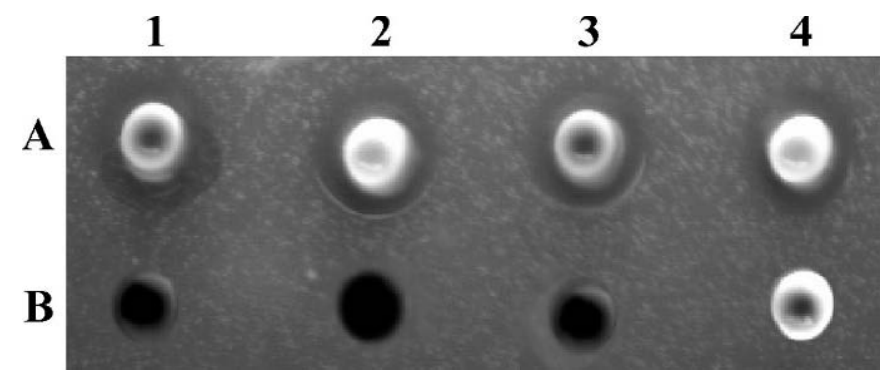

Figure 5. The antimicrobial activity assay of different secretion samples from a goat receiving injection of $\mathrm{pI}-\mathrm{bcp}-\mathrm{LfcinB} / \mathrm{bTAP}$ solution in mammary glands. A1, A2, and A3 are milk samples collected at the same time with B1 (urine), B2 (blood), and B3 (saliva), correspondingly, after injection of $\mathrm{pI}$-bcp-LfcinB/bTAP solution in left mammary glands. A4 and B4 are milk samples collected from left side and right side mammary glands injected with $\mathrm{pI}-\mathrm{bcp}-\mathrm{LfcinB} / \mathrm{bTAP}$ and pIbcp-neo solution, respectively.

and antibacterial activity assay (data not shown). However, the amount varied in different goats after $3 \mathrm{~d}$, and the amount of the expressed antibacterial peptides declined rapidly probably due to the gene being exhausted and exclusion following the milk.

The assay results of blood, urine, and saliva samples for antibacterial peptide presence compared with the milk samples are shown in Figure 5. No antibacterial activity against Staphylococcus aureus ATCC 25923 could be observed in blood, urine, saliva, and in milk samples from the right mammary gland, whereas the milk samples collected at the same time from the left mammary gland showed antibacterial activity.

\section{DISCUSSION}

Bovine mastitis is the most significant economic drain on the worldwide dairy industry. Many endeavors have been made in search of an economic and efficient therapeutic method. Concerns regarding poor cure rates, emergence of bacterial resistance, and residues in milk necessitate development of alternative therapeutic approaches to antibiotics for treatment of mastitis. One of the most interesting reports is the lysostaphin expression in the goat mammary glands (Fan et al., 2002), which utilized the metabolism product of Staphylococcus aureus to inhibit the growth of Staphylococcus aureus and exhibited bioactivity. However, the authors pointed out that the adenoviral-mediated lysostaphin expression showed $20 \%$ of the bioactivity of the recombinant prokaryotic (E. coli) form. An antibody against the lysostaphin also arose 2 wk after the gene transfer (Fan et al., 2004), which might hinder the repeated use of this protein.

Antibacterial peptides have been reported to exert antimicrobial activity against a wide range of microor- ganisms. As the sequences of the antibacterial peptides we used in this trial originated from cows, which might possess a high homology with other mammalian species, and the molecular weight is small, which may not stimulate any antibody production. Compared with other reports (Fan et al., 2004), introduction of an antibacterial peptide into a living body does not mean introduction of a foreign antigen. Therefore, repeated use of antibacterial peptide in vivo would not encounter a barrier from the viewpoint of the host defense system.

Our objective was to examine the bioactivity of antibacterial peptide expressed in mammary glands to inhibit the bacterial growth in milk. Results of this study indicated that antibacterial peptide had the potential to be an alternative or adjunct to antibiotics for intramammary infusion to treat or prevent bovine mastitis. We also demonstrated the effectiveness of plasmid-mediated transformation of mammary gland cells and the reliability in vivo to secrete bioactive antibacterial proteins. However, the concentration of the expressed peptides in the mammary glands and their effectiveness against intramammary infections needs further demonstration.

Bovine lactoferricin and bovine tracheal antibacterial peptide genes were selected for expression because they are the 2 mammalian antibacterial peptides well studied and characterized. The difference of the antibacterial activity between them and the difference of antibacterial activities against Escherichia coli $\mathrm{K}_{12} \mathrm{D}_{31}$ and Staphylococcus aureus ATCC 25923 were compared, but no significant difference could be observed from the diameter of the bacterium growth inhibition zone. More types of bacteria are expected to be tested with the expressed peptides for their antibacterial activity. The double gene expression vector carrying the LfcinB and bTAP genes was constructed to improve the yield of antibacterial peptides so that the antibacterial activity could be raised. However, the amounts of the expressed peptides between the double gene expression vector and the single gene expression vector were not shown to have an apparent difference in Tricine-SDS-PAGE (Figure 2c). The antibacterial activities between them in the bacterium growth inhibition zone test are also at a similar level (Figure 3). One possible explanation could be that the methods we used in this trial are not sensitive enough to differentiate them. Another explanation might be that the second antibacterial peptide gene (bTAP) in pI-bcp-LfcinB-bTAP vector was not properly expressed or expressed at very low level. However, this needs further demonstration in a precisely controlled experiment.

Because there was no difference between these 2 antibacterial peptides in their antibacterial activity, in practical application both antibacterial peptide genes 
can be selected as the gene of interest. However, LfcinB is derived from lactoferrin, which is one of the richest components in bovine milk, and LfcinB possesses multiple biological activities such as antiviral and antitumor activities (Di Biase et al., 2003), stimulation of adaptive immune response and antiinflammation (Feng et al., 2004), as well as antibacterial activity. Practically, it is better to adopt LfcinB than to select bTAP.

The goat $\beta$-casein promoter was used as the main control element to direct the expression of gene of interest because $\beta$-casein is one of the most abundant proteins in the milk and the protein production in the mammary gland is the most active among all kinds of organs in the body, so this promoter certainly directs gene expression in milk-specific way at lactating stage (Persuy et al., 1995), whereas it should not direct the expression of gene of interest in other tissues. Blood, urine, and saliva were also collected to submit for antibacterial activity assay, and no growth inhibition zone was observed (see Figure 5) in the assay.

For the sake of expression detection and analysis, we tried to prepare antibodies against the antibacterial peptides by repeatedly immunizing animals with similar expression vectors. However, this was unsuccessful in rabbits and mice. The reason might be that the antibacterial peptide is too small. We also linked the antibacterial peptide to egg albumin and then immunized animals, and there was no response. So, another possible reason could be that these 2 types of antibacterial peptides exist in all species. Therefore, the expression of LfcinB, bTAP, or both, in the mammary gland could not be confirmed by Western blot and other antigenantibody analysis. However, the expression of antibacterial peptides was confirmed by the following data: (1) In the SDS-PAGE, we set up strict controls including a plasmid vector control carrying no insert and a blank milk control. There was no specific band in these 2 controls. (2) The molecular weight of the expressed peptide bands is identical to that reported and estimated. (3) The antibacterial activity only presented in the milk produced by mammary glands injected with the antibacterial peptide expression plasmids; in the control plasmid and blank milk controls, there was no antibacterial activity.

The potent antibacterial activity of antibacterial peptides is well known. Their usefulness as mastitis therapeutic and preservative agent in ruminants was first put forward by our group. Although the plasmid-mediated gene transfer of antibacterial peptides highly expressed the gene transferred, the expression lasting of the transferred genes was relatively short and needs improving. Transgenic animal approach would be a clearly effective way to keep a continuous expression of such a gene. However, as the bovine eggs are re- stricted and hard to obtain and the gestation in cows is long, it would take considerable time before the strategy is actually adopted in the national dairy herd. An alternative would be to introduce a viral vector such as reported by Fan et al. (2004), although they reported that the adenoviral vector-mediated expression was not long enough. As nonreplicating retroviral vector can be packaged into infectious viral particles and keeps its infectivity, the retroviral vector inside the viral particle is able to insert into the genome of host tissue, which may confer a persistent expression, therefore to develop a retroviral-mediated gene transfer of antibacterial peptides in mammary glands would be a solution.

\section{CONCLUSIONS}

This trial demonstrated that modified antibacterial peptide genes could be introduced into goat mammary cells in vivo by a plasmid-mediated gene transfer method. Antibacterial activity of the expressed products encoded by antibacterial peptide genes was confirmed. This novel approach to combat mastitis originating from bacterial pathogens may warrant further investigation. Further study is necessary to increase the bioactivity of the antibacterial peptides and to determine the duration of expression with an appropriate retroviral-mediated mammary gland-specific expression vector, which would confer persisting protection during the susceptible period of mastitis.

\section{ACKNOWLEDGMENTS}

This project was funded by National Natural Science Foundation of China (approval no. 30471280).

\section{REFERENCES}

Aarestrup, F. M., H. C. Wegener, and V. T. Rosdahl. 1995. Evaluation of phenotypic and genotypic methods for epidemiological typing of Staphylococcus aureus isolates from bovine mastitis in Denmark. Vet. Microbiol. 45:139-150.

Archer, J. S., W. S. Kennan, M. N. Gould, and R. D. Bremel. 1994. Human growth hormone (hGH) secretion in milk of goats after direct transfer of the hGH gene into the mammary gland by using replication-defective retrovirus vectors. Proc. Natl. Acad. Sci. USA 91:6840-6844.

Bellamy, W., M. Takase, H. Wakabayashi, K. Kawase, and M. Tomita. 1992. Antibacterial spectrum of lactoferricinB, a potent bactericidal peptide derives from the $\mathrm{N}$-terminal region of bovine lactoferrin. J. Appl. Bacteriol. 73:472-479.

Bennett, R. M., K. Christiansen, and R. S. Clifton-Hadley. 1999. Estimating the costs associated with endemic diseases of dairy cows. J. Dairy Res. 66:455-459.

Castagliuolo, I., R. Piccinini, E. Beggiao, G. Palu, C. Mengoli, F. Ditadi, G. Vicenzoni, and A. Zecconi. 2006. Mucosal genetic immunization against four adhesions protects against Staphylococcus aureus-induced mastitis in mice. Vaccine 24:4393-4402.

Diamond, G., M. Zasloff, H. Eck, M. Brasseur, W. L. Maloy, and C. L. Bevins. 1991. Tracheal antimicrobial peptide, a cysteine-rich peptide from mammalian tracheal mucosa: Peptide isolation and cloning of a cDNA. Proc. Natl. Acad. Sci. USA. 88:3952-3956. 
Di Biase, A. M., A. Pietrantoni, and A. Tinari. 2003. Heparin-interacting sites of bovine lactoferrin are involved in anti-adenovirus activity. J. Med. Virol. 69:495-502.

Fan, W., K. Plaut, A. J. Bramley, J. W. Barlow, and D. E. Kerr. 2002. Adenoviral-mediated transfer of a lysostaphin gene into the goat mammary gland. J. Dairy Sci. 85:1709-1716.

Fan, W., K. Plaut, J. Bramley, J. W. Barlow, S. A. Mischler, and D. E. Kerr. 2004. Persistency of adenoviral-mediated lysostaphin expression in goat mammary glands. J. Dairy Sci. 87:602-608.

Feng, X. J., J. H. Wang, and Y. L. Yang. 2004. A review of researches on the mechanism of lactoferricin action. China Biotechnology. 24:23-26.

Gazit, E., A. Boman, H. G. Boman, and Y. Shai. 1995. Interaction of the mammalian antibacterial peptide cecropin P1 with phospholipids vesicles. Biochemistry 34:11479-11488.

Goñi, P., Y. Vergara, J. Ruiz, I. Albizu, J. Vila, and R. Gómez-Lus. 2004. Antibiotic resistance and epidemiological typing of Staphylococcus aureus strains from ovine and rabbit mastitis. Int. J. Antimicrob. Agents 23:268-272.

Harmon, R. J. 1994. Physiology of mastitis and factors affecting somatic cell counts. J. Dairy Sci. 77:2103-2112.

Heller, W. T., A. J. Waring, R. I. Lehrer, T. A. Harroun, T. M. Weiss, L. Yang, and H. W. Huang. 2000. Membrane thinning effect of the beta-sheet antimicrobial protegrin. Biochemistry 39:139-145.

Hens, J. R., M. D. Amstutz, F. L. Schanbacher, and I. H. Mather. 2000. Introduction of the human growth hormone gene into the guinea pig mammary gland by in vivo transfection promotes sustained expression of human growth hormone in the milk throughout lactation. Biochim. Biophys. Acta 1523:161-171.

Lehrer, R. I., M. Rosenman, S. S. Harwig, R. Jackson, and P. Eisenhauer. 1991. Ultrasensitive assays for endogenous antimicrobial polypeptides. J. Immunol. Methods 137:167-173.

Lohner, K., and S. E. Blondelle. 2005. Molecular mechanisms of membrane perturbation by antimicrobial peptides and the use of biophysical studies in the design of novel peptide antibiotics. Comb. Chem. High Throughput Screen. 8:241-256.

McCafferty, D. G., P. Cudic, D. C. Behenna, and R. Kruger. 1999. Synergy and duality in peptide antibiotic mechanisms. Curr. Opin. Chem. Biol. 3:672-680.

Persuy, M. A., S. Legrain, C. Printz, M. G. Stinnakre, L. Lepoury, G. Brignon, and J. C. Mercier. 1995. High-level, stage-and mammary-gland-tissue-specific expression of a caprine kappa-casein- encoding minigene driven by a beta-casein promoter in transgenic mice. Gene 165:291-296.

Sambrook, J., E. F. Fritsch, and T. Maniatis. 1989. Molecular Cloning: A Laboratory Manual, 2nd ed., Cold Spring Harbor Laboratory Press, Cold Spring Harbor, NY.

Selsted, M. E., Y. Q. Tang, W. L. Morris, P. A. McGuire, M. J. Novotny, W. Smith, A. H. Henschen, and J. S. Cullor. 1993. Purification, primary structures, and antibacterial activities of $\beta$-defensins, a new family of antimicrobial peptides from bovine neutrophils. Biol. Chem. 268:6641-6648.

Shim, E. H., R. D. Shanks, and D. E. Morin. 2004. Milk loss and treatment costs associated with two treatment protocols for clinical mastitis in dairy cows. J. Dairy Sci. 87:2702-2708.

Tomita, M., W. Bellamy, M. Takase, K. Yamauchi, H. Wakabayashi, and K. Kawase. 1991. Potent antibacterial peptides generated by pepsin digestion of bovine lactoferrin. J. Dairy Sci. 74:4137-4142.

Ulvatne, H., O. Samuelsen, H. H. Haukland, M. Kramer, and L. H. Vorland. 2004. Lactoferricin B inhibits bacterial macromolecular synthesis in Escherichia coli and Bacillus subtilis. FEMS Microbial. Lett. 237:377-384

Van't Hof, W., E. C. Veerman, E. J. Helmerhorst, and A. V. Amerongen. 2001. Antimicrobial peptides: Properties and applicability. Biol. Chem. 382:597-619.

Vogel, H. J., D. J. Schibli, W. Jing, E. M. Lohmeier-Vogel, R. F. Epand, and R. M. Epand. 2002. Towards a structure-function analysis of bovine lactoferricin and related tryptophan-and arginine-containing peptides. Biochem. Cell Biol. 80:49-63.

Wakabayashi, H., H. Matsumoto, and K. Hashimoto. 1999. N-Acylated and $\mathrm{D}$ enantiomer derivatives of anonamer core peptide of lactoferricin B showing improved antimicrobial activity. Antimicrob. Agents Chemother. 43:1267-1269.

Wang, X., and X. Y. Zhang. 2004. The situation and advance of treatment for mastitis of dairy cattle with Chinese herbal medicine. Chinese J. Vet. Drugs 38:43-46.

Watts, J. L. 1988. Etiological agents of bovine mastitis. Vet. Microbiol. 16:41-66.

Yarus, S., J. M. Rosen, A. M. Cole, and G. Diamond. 1996. Production of active bovine tracheal antimicrobial peptide in milk of transgenic mice. Proc. Natl. Acad. Sci. USA 93:14118-14121.

Zasloff, M. 2002. Antimicrobial peptides of multicellular organisms. Nature 415:389-395.

Zhu, J. M., F. P. Liu, Y. Q. Li, Z. Y. Zhong, and N. Zhang. 2002 Cloning and expression of a gene encoding shortened cecropin Amelittin hybrid in $E$. coli. Hereditas 24:31-34. 